\title{
The Influence of Hospital Type on 30-Day Emergency Department Visits After Benign Gynecologic Surgery
}

\author{
Anne M. Stachowicz, MD, ${ }^{1}$ Hoi Tik H. Yuen, MD, Deborah Levy, MD, ${ }^{2}$ \\ Gabrielle J. Farley, BS, ${ }^{1}$ and James L. Whiteside, MD, $\mathrm{MA}^{2}$
}

\begin{abstract}
Objective: The goal of this research was to examine how hospital type influences emergency department (ED) utilization within 30 days after benign gynecologic surgery.

Materials and Methods: This was a pilot retrospective cohort study. Across 2 hospital settings-an academic medical center and a community-teaching hospital — adult women undergoing benign gynecologic surgery were placed randomly into 80 subject cohorts based on their 30-day postoperative ED utilization. The cohorts were defined as: (1) no ED visit; (2) low-yield ED visit (an ED visit with same-day discharge); and (3) high-yield ED visit (an ED visit with hospital admission). An analysis of risk factors for ED use according to hospital setting was performed.

Results: Risk factors for ED use varied by hospital type. In the academic setting, Caucasian patients had fewer postoperative ED visits than patients of minority race/ethnicities, and insured patients had fewer postoperative ED visits than patients insured by Medicaid. In the academic setting, proximity to the hospital, performance of adhesiolysis, and prescription for an opiate were factors in frequency and types of ED visits. The need for catheterization was a factor in the type of ED visit only in the community-teaching setting,

Conclusions: Hospital type influences use of emergency services following benign gynecologic surgery. ( J GYNECOL SURG 37:16)
\end{abstract}

Keywords: emergency department, gynecologic surgical procedures, health facilities, postoperative timeperiod

\section{Introduction}

$\mathbf{E}$ MERGENCY DEPARTMENT (ED) overcrowding in the United States is a factor in reduced care access and quality. ${ }^{1}$ The causes of ED overcrowding are multifactorial, including visits for low-acuity problems. ${ }^{2}$ By evaluating these kinds of encounters for risk factors, it could be possible to develop strategies to reduce low-acuity ED utilization.

To investigate ED utilization within 30 days of benign hysterectomy, a statewide surgical-quality collaborative database study found that $9.1 \%$ of 10,274 women during the study time period who presented to the ED were not admitted. ${ }^{3}$ Thus, rates of postoperative ED visits not resulting in admission in other surgical specialties included $3.6 \%$ after total hip arthroplasty procedures and $6.5 \%$ after Roux-en-Y gastric-bypass procedures. ${ }^{4,5}$ Approximately one-third of the posthysterectomy visits were classified as potentially avoidable, based on the presenting chief complaints. ${ }^{3}$ Risk factors for avoidable ED visits included younger age, Medicaid insurance, and higher postoperative day-1 pain scores. Although that study identified risk factors for potentially avoidable postoperative ED visits, cases from both academic and community institutions were included in the sample with no differential analysis by hospital types.

A systematic review of models estimating the risk of hospital readmission emphasized that, among other factors, consideration must be given to the settings and populations that the models used. ${ }^{6}$ The objective of the current study was to compare patient and perioperative characteristics between low-yield and high-yield ED visits within 30 days of benign gynecologic surgery in 2 different hospital settings.

\footnotetext{
${ }^{1}$ Female Pelvic Medicine and Reconstructive Surgery, The Christ Hospital, Cincinnati, Ohio, USA.

${ }^{2}$ Department of Obstetrics and Gynecology, University of Cincinnati College of Medicine, Cincinnati, Ohio, USA.

Presented as a poster at the Society of Gynecologic Surgeons 45th Annual Scientific Meeting, in Tucson, AZ, USA, March 31-April 3, 2019.
} 


\section{Materials and Methods}

\section{Sample}

A retrospective chart review was performed of the electronic medical records (EMRs) of women who underwent scheduled benign gynecologic surgery at either an academic medical center or a community-teaching hospital between 2011 and 2019. Both hospitals are in an urban setting. The academic medical center has 550 staffed beds and is associated with a university-based medical school. The communityteaching hospital has 538 staffed beds, and medical students and postgraduate trainees from the medical school rotate through this hospital. A total of 40 subjects per cohort per hospital site was deemed to be a sufficient sample for this pilot investigation designed to examine the effect of hospital setting on the ability to predict postoperative ED visits. The bioinformatics department of each hospital used computerized randomization to place subjects meeting search criteria into 3 cohorts of 40 subjects each.

In line with a prior study, ${ }^{3} \mathrm{ED}$ visits without hospital admissions included instances that were potentially avoidable and could accordingly be regarded as "low-yield." Conversely, ED visits with hospital admissions could be regarded as "highyield." Therefore, 3 cohorts were defined as: (1) no ED visit; (2) low-yield ED visit (an ED visit with same-day discharge to go home); and (3) high-yield ED visit (an ED with hospital readmission). Benign gynecologic surgery was defined as any gynecologic procedure with benign preoperative and final pathologic diagnoses, and included a variety of procedures from hysteroscopy to hysterectomy. Emergent cases, subjects $<18$ years of age, and pregnancy-related or oncologic cases were excluded. Within the study metropolitan service area, all major health systems rely on a single EMR maker (Epic Systems Corporation, Verona, WI, USA) with shared patienthealth information via the regional Health Information Exchange. In this way, it was assumed that nearly all ED visits could be captured within the 30-day postoperative period. For subjects who had multiple ED visits, data were collected from the first ED visits and, if any ED visits resulted in readmissions, they were excluded from the low-yield cohort.

Demographic data points collected included race, age, body mass index (BMI), insurance carrier, county and state of residency, medical comorbidities (including history of chronic pain or venous thromboembolism), current tobacco use, and number of ED visits in the previous year. An estimate of cumulative risk attributable to comorbid conditions was performed using the Charlson comorbidity index ${ }^{7}$ as reported by Quan et al. ${ }^{8}$ ED visits within the 365 days prior to the index surgery were counted to obtain the number of ED visits in the previous year.

Perioperative data points collected included date of surgery, preoperative diagnosis, procedure performed, operative time, mode of anesthesia (monitored anesthesia care, laryngeal mask airway, or endotracheal tube airway, American Society of Anesthesiologists (ASA) class, attending type (obstetrics/gynecology generalist/subspecialist), need for intraoperative surgical route conversion, length of hospital stay, presence of intra- or immediate postoperative complications, need for catheterization upon discharge, type of discharge instructions (generic, procedure-specific, or specialty-specific), discharge prescription for opiate received, ED visit chief complaint, and disposition from ED visit. Dates of surgery were categorized into 4 academic "seasons" with July 1st as the start of a new academic year.

Preoperative diagnoses were categorized into 2 groups based on the presence/absence of a pain-related diagnosis as the indication for surgery (e.g., dysmenorrhea, pelvic pain, etc.). Procedures were categorized by minor versus major, with major procedures subcategorized further by route, (i.e., abdominal, laparoscopic (including robotic), and vaginal). Minor procedures were defined as any intraperitoneal procedures lasting $\leq 30$ minutes and all extraperitoneal procedures. Cases were considered affirmative for adhesiolysis if the operative report listed adhesiolysis in the procedures-performed section of the form. Surgical complications were assigned a severity level based on the modified Expanded Accordion Severity Grading System for surgical complications described by Albright et al. ${ }^{9}$ Discharge instructions listed in the electronic discharge summary were categorized as generic, procedurespecific, or specialty-specific based upon the title (e.g., "Laparoscopic Hysterectomy Instructions"), and a subjective assessment of how the instructions were-or were not-tailored to the case at hand. Information regarding opiate prescriptions was obtained from the discharge summaries.

\section{Data analysis}

Preliminary frequency analyses were performed across the variables collected relative to ED disposition. Bivariate analyses were performed using $\chi^{2}$ or Fisher's exact tests for categorical variables and analysis of variance (ANOVA) for continuous variables. Post-hoc analyses included pairwise comparisons with Bonferroni correction for $\chi^{2}$, and Fisher's exact tests and Tukey Honestly Significant Difference (HSD) for ANOVA were performed.

All analyses were performed using SPSS Statistics, version 1.0.0.1275 (IBM, Armonk, NY, USA). All statistical tests were 2 -sided with $p<0.05$ regarded as the significance threshold.

\section{Ethics approvals}

Two approvals were obtained. The Christ Hospital, in Cincinnati, $\mathrm{OH}$, was the institutional review board (IRB) of record (IRB \#:16-29; date of approval: September /3, 2019). The University of Cincinnati, in $\mathrm{OH}$, was the ancillary IRB (IRB \#: 2016-5754; date of approval: July 30, 2019).

\section{Results}

The study cohort consisted of 240 subjects ( 3 groups of 80 subjects) who had undergone surgery from 2011 to 2019. The subjects' mean age was 45.9 years (standard deviation [SD] 12.5). Study patients were $56 \%(n=135)$ Caucasian, $40 \%$ $(n=97)$ African American, 2\% $(\mathrm{n}=4)$ Hispanic, $1 \%(n=2)$ Asian, and 1\% $(n=2)$ other/not specified. The mean BMI of participants was $31.0 \mathrm{~kg} / \mathrm{m}^{2}$ (SD 8.4). The patient population's insurance carriers consisted of $48 \% \quad(n=116)$ private, 34\% $(n=82)$ Medicaid, 14\% $(n=34)$ Medicare, and $3 \%(n=8)$ self-pay/uninsured. Eighty-eight percent $(n=210)$ lived in-state, and $65 \%(n=155)$ lived in-county. Sixty-three percent $(n=150)$ had no significant comorbidities, $32 \%(n=77)$ had 1 comorbidity, and 5\% $(n=13)$ had comorbidity scores of 2 or more. Most patients did not have diagnoses of chronic pain $(n=57,24 \%)$ or histories of venous thromboembolism 
$(n=21,8 \%)$. Most patients were nonsmokers $(n=181,75 \%)$. The mean number of ED visits in the year prior to surgery was 2.1 (SD 4.6).

In overall analyses inclusive of both hospital types, postoperative ED utilization was associated with race/ethnicity $(p=0.04)$, ASA class $(p=.01)$, insurance type $(p<0.01)$, county of residency $(p=0.04)$, academic season $(p=0.02)$, operative time $(p<0.001)$, mode of anesthesia $(p<0.001)$, attending specialty $(p=0.01)$, route of surgery $(p<0.001)$, length of stay $(p<0.001)$, complication-severity grade $(p<0.001)$, discharge with catheterization $(p=0.001)$, opiate prescription distribution $(p<0.001)$, comorbidity index severity $(p=0.03)$, and ED visits in the year prior to surgery $(p<0.001)$.

In a subanalysis by hospital type, however, differences were identified (Table 1). In the academic setting, a relationship persisted between ED utilization and race/ethnicity, ASA class, insurance type, county of residency, academic season, operative time, mode of anesthesia, attending specialty, route of surgery, length of stay, complication-severity grading, opiate prescription distribution, comorbidity-index severity, and ED visits in the year prior to surgery. In contrast, in the community-teaching hospital, only the association of ED utilization with operative time, mode of anesthesia, attending specialty, route of surgery, length of stay, complication-severity grade, and urinary-catheter requirement at discharge persisted.

No relationship could be established between postoperative ED utilization and state of residence, pain-related surgical indication, performance of lysis of adhesions, or discharge instruction-specificity when analyzed inclusive of hospital type. Analysis relative to hospital type revealed that postoperative ED utilization was associated with state of residence, pain-related surgical indication, and performance of lysis of adhesions only in the academic setting. In addition, in the community-teaching setting, a relationship between ED utilization and discharge instruction-specificity was identified.

Post-hoc pairwise comparisons of hospital-specific bivariate tests enabled a more granular understanding of the relationship of the study variables to ED utilization. For example, in the academic setting, relative to the null model, Caucasian patients had fewer postoperative ED visits than patients of minority race/ethnicities. Likewise, privately insured patients had fewer postoperative ED visits than patients insured by Medicaid. While analysis inclusive of hospital type for state of residence was not statistically significant, in the academic setting, patients living out of state visited the ED less often and in-state patients visited the ED more often than expected in the null model. While cumulative frequency analysis for ED visits after performance of adhesiolysis was not statistically significant, in the academic setting, patients requiring adhesiolysis had more high-yield ED visits and patients not requiring lysis of adhesions had fewer high-yield ED visits than the null model expected. Finally, in the academic setting only, patients who did not visit the ED more often had received prescriptions for opiates, and patients who had highyield visits also had received opiate prescriptions more often than the null model predicted. Only in the communityteaching setting, patients who could self-void upon discharge had fewer low-yield ED visits and patients who were discharged needing catheterization had more low-yield ED visits then expected in the null model.

\section{Discussion}

Hospital context does appear to influence the kind and frequency of ED visits among women undergoing benign gynecologic surgery. Our pooled analyses, irrespective of hospital type, aligned with previously identified risk factors, including race, ASA class, insurance type, length of stay, presence of preoperative comorbidity, presence of complications, and previous ED visit frequency. ${ }^{3,10} \mathrm{New}$ potential risk factors not previously reported include proximity to hospital, academic season, operative time, mode of anesthesia, attending specialty, route of surgery, discharge with bladder catheterization, and dispersal of opiate prescriptions.

Some relationships between demographic and perioperative variables and postoperative ED utilization did change when analyzed by hospital type. For example, insurance type was associated with ED utilization in pooled analyses; however, when analyzed by hospital setting, Medicaid status was associated with ED utilization only in the academic medical center setting. Likewise, discharge needing bladder catheterization was associated with ED utilization in pooled analyses; however, only in the community-teaching setting was the ability to self-void upon discharge associated with less low-yield ED utilization.

The wide variation in patient-, clinician-, and hospitallevel factors between the 2 hospital settings demonstrated by the current study supports the concept that determinants of risk between hospitals might be different. Kansagara et al. ${ }^{6}$ postulated that, while medical comorbidities might be the predominant risk factors in 1 population, social determinants, such as housing type, might be a larger source of risk for an alternative setting. Accordingly, application of a risk model focusing on patient- and clinical-level characteristics to both settings could yield an inaccurate prediction, particularly, in the population for which the greatest risk is contributed by social-level factors.

The few studies examining unplanned 30-day hospital visits after benign gynecologic surgery ${ }^{3,10}$ failed to include hospitallevel or social factors and, instead, focused on patient- and clinical-factors. Borrowing research from other surgical theaters, psychosocial and hospital-based factors affected rates of ED recidivism and readmissions. ${ }^{11,12}$ Inclusion of these types of factors in the development of models from retrospective cohort studies can be problematic, as their documentation in databases and/or EMRs is uncommon. Furthermore, existing risk models were designed for easy real-time application by utilizing data readily available to care providers. Thus, incorporation of social-determinants, (e.g., neighborhoodlevel socioeconomic status) might make model use impractical. Ideally, a model to predict low-yield ED utilization would incorporate all facets of risk accounting for the idiosyncrasies among the populations served by different hospitals. Once such risk factors are realized, mitigation strategies can be created. As with politics, arguably, all ED visit patterns are local.

Strengths of this study included a random sample of cases of benign gynecologic surgery from both an academic medical center and a community hospital, broadening the generalizability of the results. In addition, given a robust Health Information Exchange, nonrecorded ER visits were limited. Limitations of this study are that the cohort size was small in comparison with previous studies and that the only 


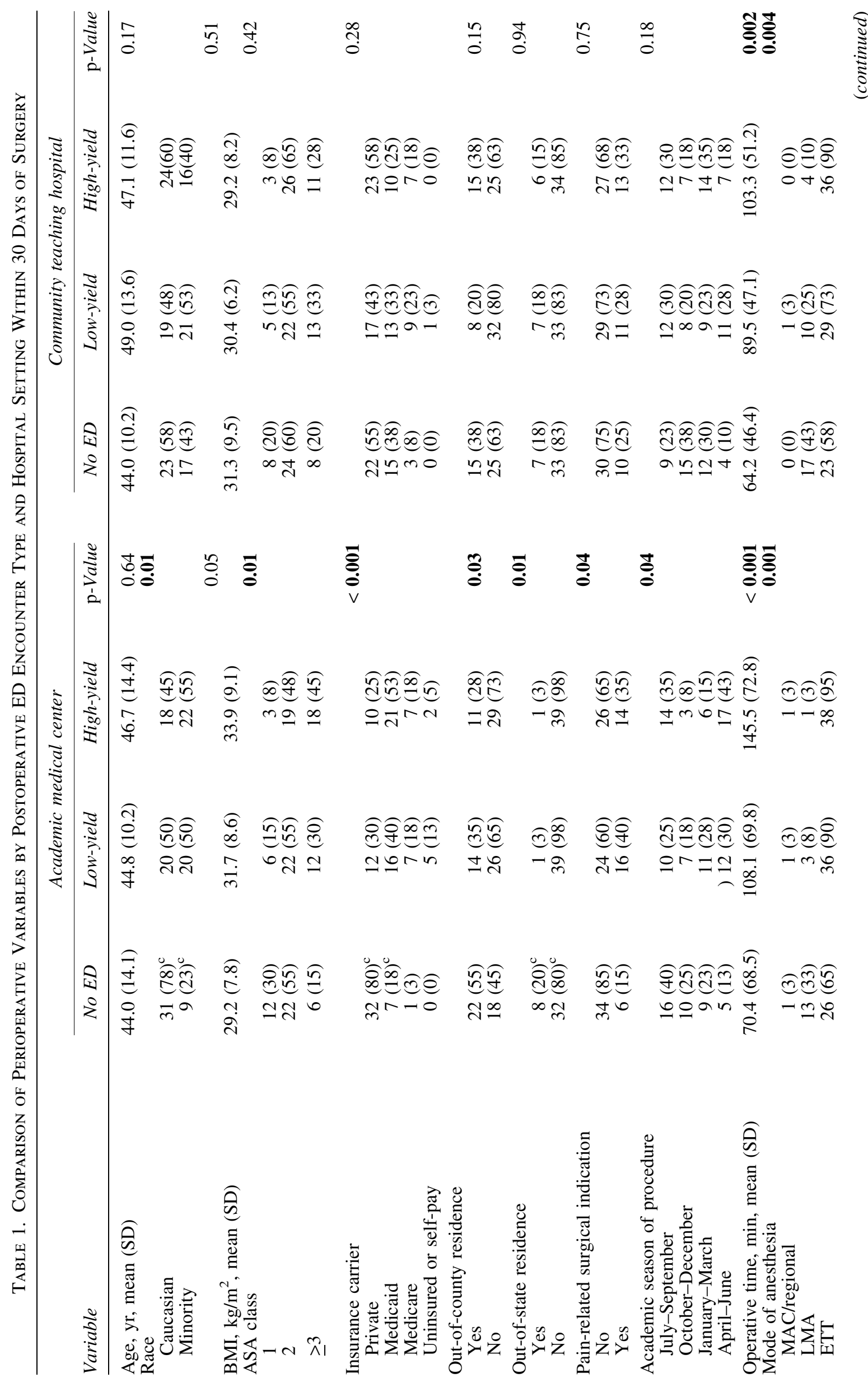




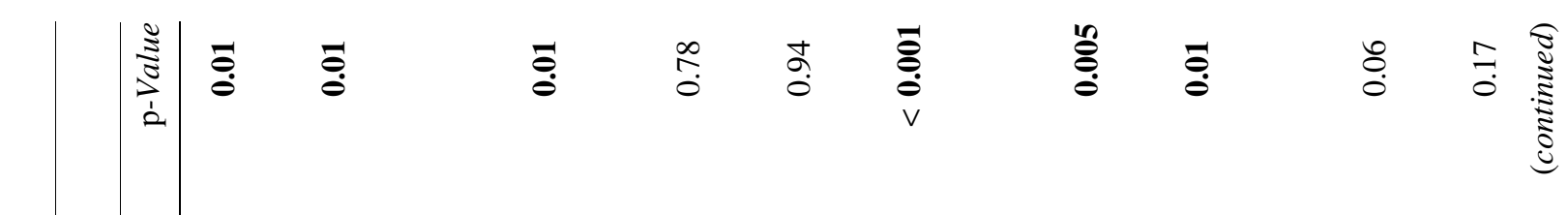

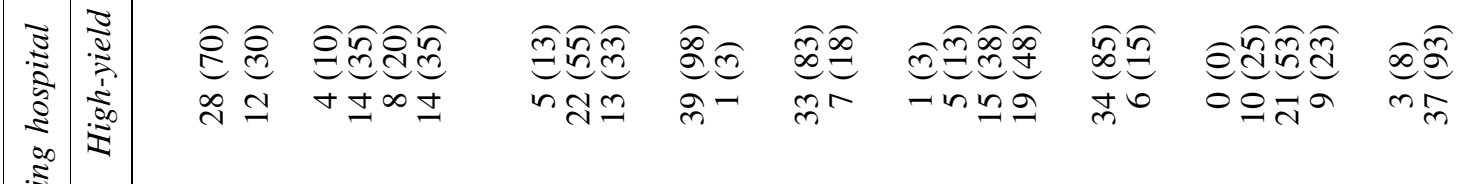

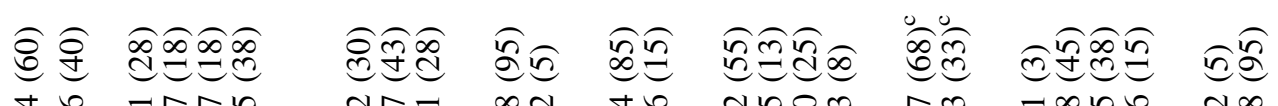

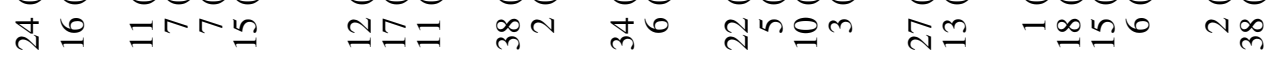

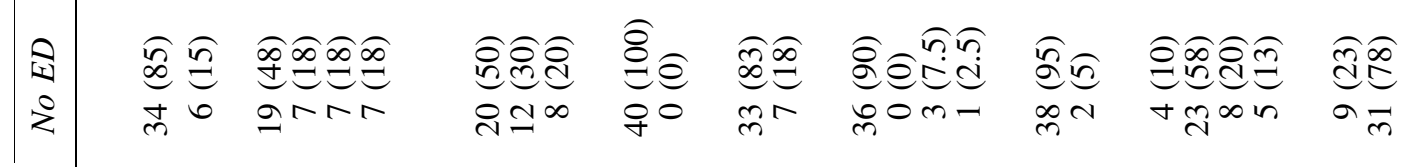

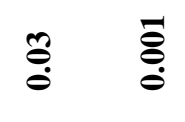

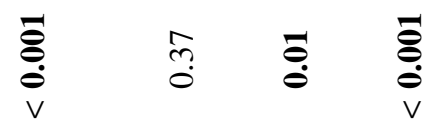
$\stackrel{n}{0}$

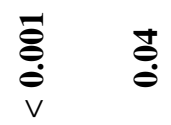

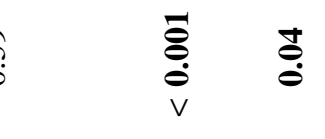

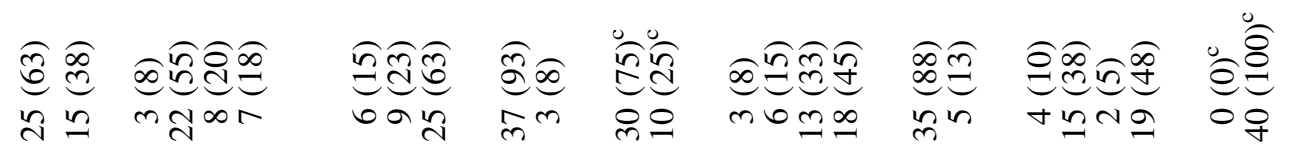

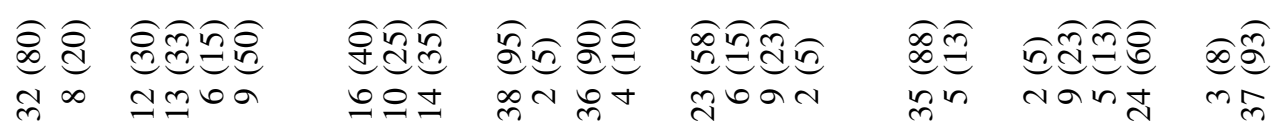

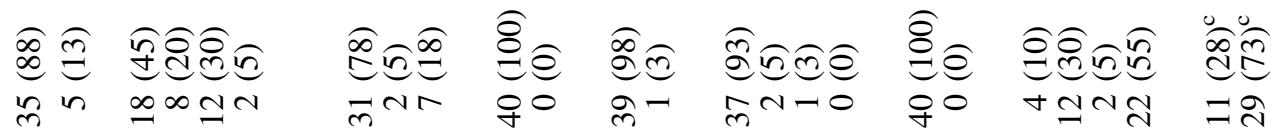

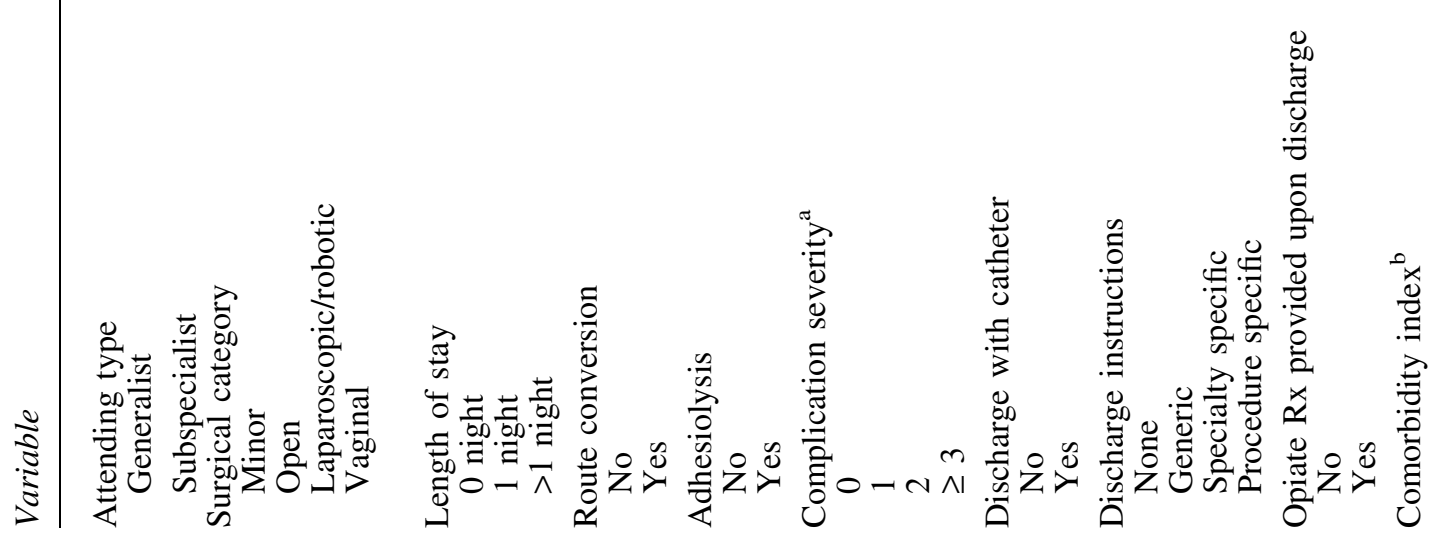




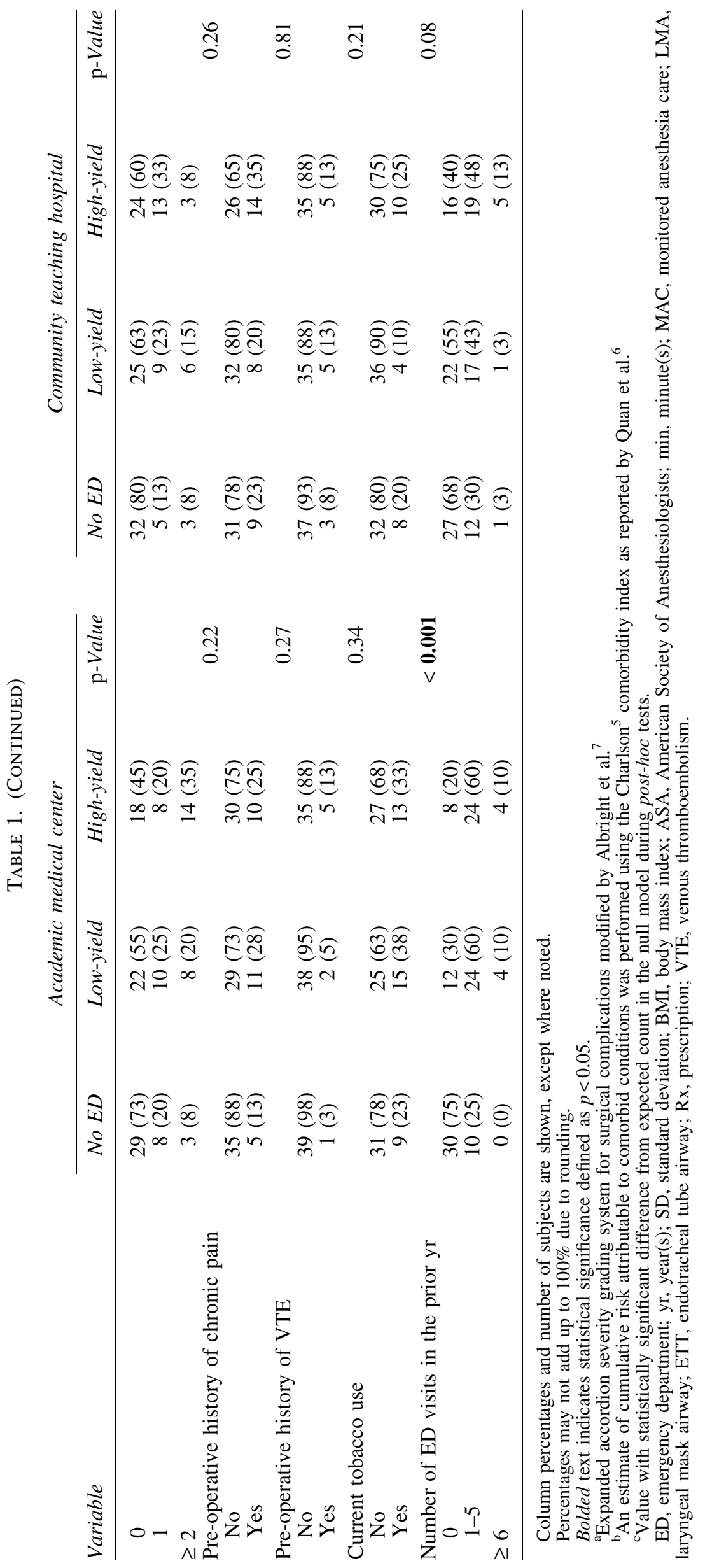


patient-hospital interactions included were those that were recorded in the available EMR system. Furthermore, it was assumed that all ED physicians have similar thresholds to admit patients for more-severe illness and manage as outpatients those who are less ill. Complications that were managed in alternative settings (e.g., urgent care or other non-EMR-sharing emergency departments) could have been unavoidably missed. Handwritten opiate prescriptions or discharge instructions might have not been captured accurately by the EMR. In addition, there was no way to confirm whether opiate prescriptions were ultimately filled or not.

Although current understanding of risk factors for postoperative ED utilization helps identify at-risk patients who could benefit from additional pre- or postoperative education or outpatient visits, future investigations are needed to adjust such analyses of risk for hospital-level factors, as this pilot study suggests these could influence postoperative ED utilization significantly. In addition, until such research is available, practitioners should keep their practice settings in mind when applying current risk-reduction models.

\section{Conclusions}

Strategies to mitigate ED utilization among postgynecologic surgery patients should consider the hospital context.

\section{Acknowledgments}

The current authors thank Suma Reddy, MD, and Patrick Lang, MD, their contributions to this article.

\section{Author Disclosure Statement}

James L. Whiteside, MD, MA, was paid by the American Urogynecologic Society, the American College of Obstetrics and Gynecologists, and Legal Reviews. No other financial conflicts of interest exist.

\section{Funding Information}

There was no financial support for this research.

\section{References}

1. Trzeciak S, Rivers EP. Emergency department overcrowding in the United States: An emerging threat to patient safety and public health. Emerg Med J 2003;20:402.
2. Schiff GD. System dynamics and dysfunctionalities: Levers for overcoming emergency department overcrowding. Acad Emerg Med 2011;18:1255.

3. Mahnert N, Kamdar N, Lim CS, et al. Risk factors for emergency department visits after hysterectomy for benign disease. Obstet Gynecol 2017;130:296.

4. Saleh A, Faour M, Sultan AA, Brigati DP, Molloy RM, Mont MA. Emergency department visits within thirty days of discharge after primary total hip arthroplasty: A hidden quality measure. J Arthroplasty 2019;34:20.

5. Kellogg TA, Swan T, Leslie DA, Buchwald H, Ikramuddin S. Patterns of readmission and reoperation within 90 days after Roux-en-Y gastric bypass. Surg Obes Relat Dis 2009;5:416.

6. Kansagara D, Englander H, Salanitro A, et al. Risk prediction models for hospital readmission: A systematic review. JAMA 2011;306:1688.

7. Charlson ME, Pompei P, Ales KL, MacKenzie CR. A new method of classifying prognostic comorbidity in longitudinal studies: Development and validation. J Chronic Dis 1987;40:373.

8. Quan H, Sundararajan V, Halfon P, et al. Coding algorithms for defining comorbidities in ICD-9-CM and ICD-10 administrative data. Med Care 2005;43:1130.

9. Albright BB, Witte $\mathrm{T}$, Tofte $\mathrm{AN}$, et al. Robotic versus laparoscopic hysterectomy for benign disease: A systematic review and meta-analysis of randomized trials. J Minim Invasive Gynecol 2016;23:18.

10. Glauser G, Winter E, Caplan IF, et al. Composite score for outcome prediction at 30 days in gynecologic surgery patients. J Healthc Qual 2020; March 4; e-pub ahead of print.

11. Kurtz SM, Lau EC, Ong KL, Adler EM, Kolisek FR, Manley MT. Hospital, patient, and clinical factors influence 30- and 90-day readmission after primary total hip arthroplasty. J Arthroplasty 2016;31:2130.

12. Weissman JS, Stern RS, Epstein AM. The impact of patient socioeconomic status and other social factors on readmission: A prospective study in four Massachusetts hospitals. Inquiry 1994;31:163.

Address correspondence to: Anne Stachowicz, MD

Female Pelvic Medicine and Reconstructive Surgery

The Christ Hospital

2123 Auburn Avenue, Suite 307 Cincinnati, OH, 45219

USA

E-mail: anne.stachowicz@thechristhospital.com 